\title{
Lasers à électrons libres de courtes longueurs d'onde : état de l'art et perspectives
}

\author{
M.E. Couprie ${ }^{1,2}$ \\ ${ }^{1}$ Service Photons, Atomes et Molécules, CEA/DSM/DRECAM, bâtiment 522, \\ 91191 Gif-sur-Yvette, France \\ ${ }^{2}$ Laboratoire pour I'Utilisation du Rayonnement Electromagnétique, LURE, bâtiment 209D, \\ BP. 34, Centre Universitaire Paris-Sud, 91898 Orsay cedex, France
}

\begin{abstract}
Les Lasers à Électrons Libres (LEL) sont des sources de rayonnement cohérent et accordable, basées sur l'interaction d'un faisceau d'électrons relativistes circulant dans le champ magnétique magnétique périodique et permanent créé par un «onduleur», et d'une onde optique. L'onde lumineuse peut être jssue de l'émission de rayonnement synchroton émise par le paquet d'électrons à chaque passage pour un système super-radiant en mode SASE (Self Amplified Spontaneous Emission- Émission Spontanée Auto-Amplifiée) ou issue du rayonnement synchrotron stocké dans une cavité optique, en mode oscillateur, ou d'une onde laser externe, en mode génération d'harmonique. Sous certaines conditions, l'onde lumineuse est amplifiée au détriment de l'énergie cinétique des électrons, ce qui conduit à l'effet laser. L'état de l'art des sources Laser à Électrons Libres dans l'UV et le VUV est présenté, en indiquant les complémentarités résultant des différentes configurations tant du point de vue des sources que de leurs utilisations. Les projets de sources de courte longueur d'onde proposés sont ensuite discutés.
\end{abstract}

\section{INTRODUCTION}

Dans le cas d'un LEL [1], les particules relativistes d'énergie normalisée $\gamma$ sont accélérées transversalement suite au champ magnétique de l'onduleur, elles émettent donc du rayonnement synchrotron dont les caractéristiques spectrales dépendent du champ magnétique (amplitude $\mathrm{B}_{\mathrm{O}}$, période $\lambda_{\mathrm{O}}$ ) et de l'énergie normalisée des particules $\gamma$. Le rayonnement synchrotron sur l'axe est émis à la

longueur d'onde $\lambda$, dite de résonance, et ses harmoniques impaires, sur l'axe : $\quad \lambda=\frac{\lambda_{0}}{2 \gamma^{2}}\left(1+\frac{K^{2}}{2}\right)$ où $\mathrm{K}$ désigne le paramètre de déflexion de l'onduleur, soit $\mathrm{K}=0.94 \lambda_{\mathrm{O}}(\mathrm{cm}) \mathrm{B}_{\mathrm{O}}(\mathrm{T})$. L'interaction de cette onde optique avec le paquet d'électrons se réalise au fur et à mesure de la progression dans l'onduleur, et se produit généralement sur de multiples passages lorsqu'une cavité optique de longueur adaptée à la récurrence des paquets d'électrons permet de stocker le rayonnement. Un échange d'énergie a lieu entre l'onde optique et les particules chargées, pouvant conduire à une modulation de la densité électronique des électrons à la longueur d'onde $\lambda$, mettant ainsi en phase l'émission et renforçant la cohérence du rayonnement produit. Ce principe est analogue à celui des klystrons micro-ondes: la modulation (en énergie puis spatiale) du faisceau d'électrons s'apparente à celle produite dans les cavités résonnantes du klystron, conduisant à la mise en cohérence des émetteurs, et à l'émission spontanée. Dans le cas du LEL, un échange supplémentaire d'énergie au deuxième ordre entre l'onde optique et le faisceau d'électrons conduit à l'amplification de l'onde lumineuse stockée dans la cavité optique qui voit alors, au fur et à mesure des interactions, son intensité augmenter de façon non linéaire jusqu'à atteindre la saturation où le gain du système devient égal aux pertes du résonateur optique, et sa largeur spectrale et temporelle s'affiner. L'accordabilité du laser, l'un des atouts majeurs du LEL, s'obtient, à l'intérieur de la gamme spectrale fixée par l'énergie, par simple modification du champ magnétique de l'onduleur: limitée par les possibilités de l'onduleur, elle est d'autant plus grande que le gain est élevé ę que la bande passante des miroirs est large. 


\subsection{Configurations de Laser à Électrons Libres}

La configuration la plus souvent utilisée, l'oscillateur (cf fig. 1a), permet de stocker l'impulsion lumineuse afin que l'interaction électrons photons se produise sur des passages successifs. Cette configuration est employée de façon usuelle pour les LELs serveurs pour les applications. Une variante de cette configuration, connue sous le nom de MOPA (Master Oscillator Power Amplifier), mise en auvre à Stanford [2], consiste à mettre, à la suite du premier oscillateur, un onduleur permettant d'amplifier le rayonnement laser produit, sans trop dégrader les optiques et en fournissant ainsi un laser de puissance.

La deuxième configuration est celle de la génération d'harmoniques cohérentes (GHC) (cf fig. 1b). Elles sont produites par interaction avec le faisceau dans l'onduleur en un seul passage, à partir d'une source laser extérieure. Le phénomène de groupement en micro-paquets dû à l'onde électromagnétique se produit aussi sur les harmoniques de la longueur d'onde de résonance [3]. Les harmoniques cohérentes peuvent aussi être générées par le LEL lui-même. La cohérence des harmoniques peut aussi être renforcée à l'aide d'un onduleur adapté, dont le fondamental rayonné serait ajusté sur l'harmonique à amplifier (on peut à cet effet utiliser un onduleur de période sub-harmonique ou réduire le champ magnétique).

Dans le mode SASE, le gain du système est suffisamment important pour disposer d'une onde cohérente à la fin de l'onduleur, qui est en général très long (voir fig. 1c) [4].
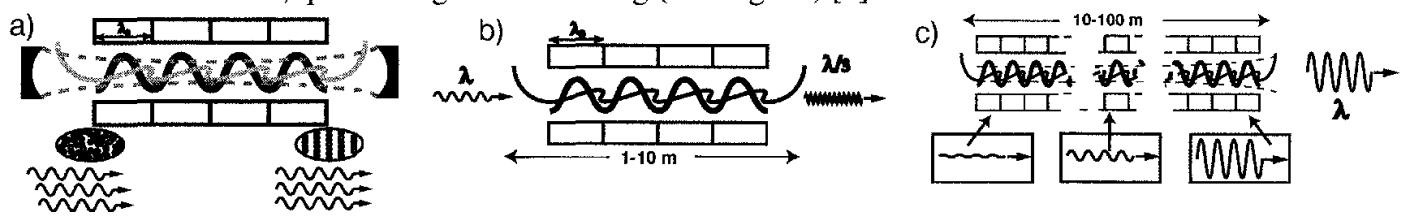

Figure 1. Diagrammes schématiques des configurations LEL a) oscillateur, b) génération d'harmoniques cohérentes c) SASE

\subsection{Choix de l'accélérateur}

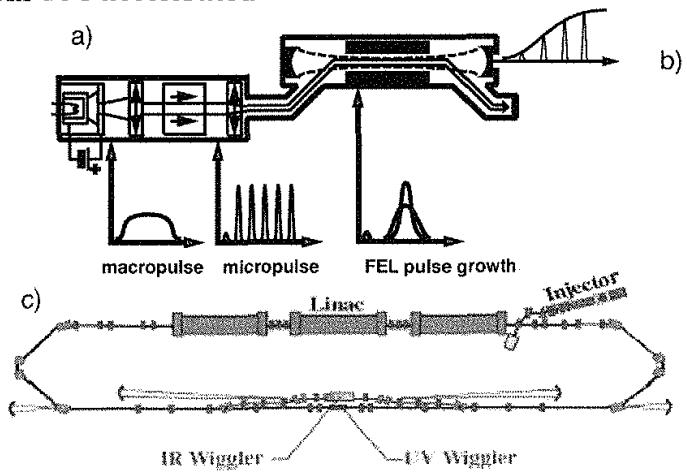

b)

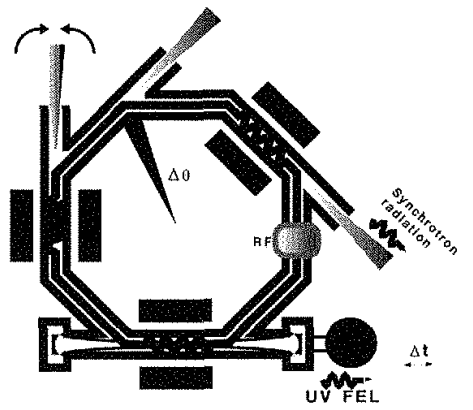

Figure 2. Diagramme des différents types d'accélérateur pour le LEL a) LINAC, b) anneau de stockage c) ERL

La gamme spectrale dépend de l'énergie des particules, et donc du type d'accélérateur. Ainsi, des accélérateurs de basse énergie (de l'ordre du MeV) conduisent au domaine micro-onde, d'énergie intermédiaire $(50 \mathrm{MeV})$ à l'infrarouge moyen et à l' U.V., et d'énergie plus élevée (GeV) à l'U.V., VUV et aux rayons X. Pour les LELs de courte longueur d'onde, le faisceau d'électrons relativistes est issu d'un accélérateur linéaire (LINAC) (voir fig.2a) ou d'un anneau de stockage (AS) (voir fig. 2b). Sur LINAC, le faisceau d'électrons est perdu à la fin et un paquet "neuf" interagit avec le LEL à chaque passage. Le faisceau d'électrons se présente sous forme de macro-impulsions d'une dizaine de $\mu$ s contenant un certain nombre de micro-impulsions étroites et à haute cadence. Des macro-impulsions nettement plus longues peuvent être délivrées par des accélérateurs linéaires supraconducteurs. Sur anneau de stockage, le faisceau recircule sur un grand nombre de tours grâce à une cavité radio-fréquence permettant, à l'aide d'un champ électrique accélérateur, de ré-accélérer les particules à chaque tour pour leur redonner l'énergie qu'elles ont perdu par rayonnement synchrotron. Le laser reproduit la structure pulsée à l'échelle du $\mathrm{MHz}$ délivrée par les paquets d'électrons. Un intérêt croissant est maintenant porté sur les ERL (Energy Recovery LINAC), LINAC à récupération d'énergie, où le faisceau est décéléré et réaccéléré par les mêmes structures accélératrices (voir fig. 2c) [5]. 


\section{OSCILLATEUR ET GHC SUR LINAC ET ANNEAU DE STOCKAGE}

\subsection{Oscillateur et GHC sur anneau de stockage}

Cinq LELs sur anneau de stockage fonctionnent actuellement : Super-ACO en France [6], ELETTRA (collaboration européenne) en Italie [7], NIJI-4 [8] et UVSOR [9] au Japon, DUKE [10] aux USA, et ils ont montré un régime «CW à l'échelle de la $\mathrm{ms}$, outre la répétition à haute cadence des micro-impulions $(\mathrm{MHz})$. Des systèmes de contre-réaction longitudinale furent mis en œuvre sur les LELs de Super-ACO [11] et de UVSOR [12], réduisant les fluctuations d'intensité à $1 \%$ et compensant le jitter du laser. Une contre-réaction transverse est aussi en place sur le laser de DUKE [13]. Les LELs sur anneau de stockage fonctionnement quasiment à la limite de Fourier, avec des impulsions de quelques ps (FWHM) et des largeurs spectrales relatives étroites (0.1-0.01\%), appropriées aux études spectroscopiques. La gamme spectrale couvre le domaine UV, et VUV sur les LELs de DUKE et d' ELETTRA, qui détient le record de la plus courte longueur d'onde pour un LEL en mode oscillateur à $190 \mathrm{~nm}$. Des puissances moyennes supérieures au Watt sont maintenant atteintes, comme à UVSOR [14]. Des expériences d'utilisation des LELs sur anneau de stockage sont maintenant en œuvre sur Super-ACO depuis 1993 [15], à DUKE depuis 2000 [16], à ELETTRA et à UVSOR [14] depuis 2001 dans différents domaines scientifiques. Néanmoins, les performances ultimes ne peuvent être atteintes que sur un anneau de troisième génération possédant de très bonnes qualités de faisceau, et disposant d'une section droite longue pour l'élément d'insertion. Tandis qu'ELETTRA satisfait la première condition et DUKE la seconde, SOLEIL reste le seul anneau de stockage pouvant offrir des performances plus ultimes pour un LEL sur anneau de stockage [17]. Des puissances de quelques dizaines de Watt sont donc attendues.

La génération d'harmoniques cohérentes a permis de détenir pendant une dizaine d'année le record de la plus courte longueur d'onde avec un système basé sur l'interaction LEL, avec la production des harmoniques 3 et 5 d'un laser Yag doublé sur Super-ACO [18]. Il est maintenant possible d'envisager de couvrir le domaine VUV jusqu'à $30 \mathrm{~nm}$ avec des systèmes Titane: Saphir d'impulsions $100 \mathrm{fs}$ à une cadence du kHz. La GHC à partir du LEL lui-même peut être envisagée dans certains cas.

\subsection{Oscillateur et GHC sur LINAC}

Fonctionner en oscillateur avec un accélérateur linéaire permet de s'affranchir de la limite de puissance à une fraction de la puissance rayonnée par rayonnement synchrotron (la «limite de Renieri» [19]) comme dans le cas des anneaux de stockage, et des puissances moyennes peuvent être atteintes. De même, les LINACs peuvent offrir des paquets d'électrons sub-ps, ce qui conduit à un LEL fs, ce qui est attrayant pour certaines expériences résolues en temps. La GHC est aussi tout à fait exploitable [20]. Le projet SPARC en Italie prévoit de tester ces différentes configurations pour couvrir le domaine UV à X mou avec des impulsions fs. Il sera ensuite continué par le projet FERMI (Trieste) ou SPARX (ENEA-INFN) dans le domaine $\mathrm{X}$.

\section{SOURCES SASE IMPLANTÉES SUR ACCÉLÉRATEUR LINÉAIRE}

Le SASE fut proposé pour s'affranchir des difficultés liées aux optiques en réalisant l'émission cohérente en un seul passage. Dans le domaine $X$, ceci nécessite des faisceaux d'électrons aux performances ultimes : courant d'électrons d'intensité crête très élevée ( $>1 \mathrm{kA})$, divergence très faible du faisceau d'électrons (de l'ordre de $0.1 \mu \mathrm{rad}$ ) et onduleur très long, jusqu'à une centaine de mètres. Les accélérateurs linéaires sont les mieux adaptés pour fournir les performances compatibles avec le SASE, en particulier pour le domaine $\mathrm{X}$ où l'énergie des faisceaux doit être de 10 à $20 \mathrm{GeV}$ ce qui rend l'accélérateur très coûteux. Un des points les plus délicats reste le canon à électrons : l'extraction des électrons se fait non plus par chauffage mais par excitation avec un faisceau laser intense et les électrons sont accélérés à l'aide d' un champ radiofréquence de gradient élevé (50 MeV/m). Après l'observation de la saturation du SASE dans l'infra-rouge proche [21], LEULT (Argonne, USA) l'a obtenue à 530 et 385 nm [22], TESLA-TTF (DESY, Allemagne) dans la gamme 125-85 nm [23] et DUVFEL (Brokhaven) à $400 \mathrm{~nm}$ [24]. D'autres projets sont en cours: SLAC (Stanford, USA) SCSS (Spring-8, Japon) ou en discussions : à Bessy (Allemagne), en Suède et en Italie.

La divergence angulaire du rayonnement est bien plus faible que celle de l'onduleur et assure une bonne cohérence spatiale. La cohérence temporelle est partielle, avec des impulsions de $50 \mathrm{fs}$ et une distribution spectrale présentant plusieurs raies. Une limitation essentielle inhérente au processus de SASE lui-même vient du fait que l'émission est générée à partir du bruit, et qu'elle présente des 
« spikes » dont on ne contrôle ni l'intensité ni la position temporelle. Des systèmes de compensation sont proposés, mais non testés à ce jour. De plus, il y a un jitter entre l'impulsion SASE principale et l'impulsion du laser irradiant la photocathode de l'injecteur, suite à des dérives dans les sections de compression d'impulsion accélérateur, et suite au processus même basé sur le bruit. Un effort particulier de R\&D (synchronisation à $200 \mathrm{fs}$ visée dans 3 ans) reste à faire pour pouvoir exploiter réellement la structure temporelle fs pour des expériences pompe-

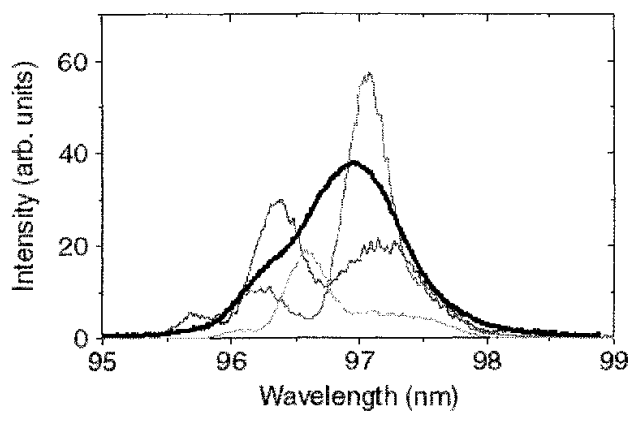
sonde à deux couleurs, couplant l'impulsion SASE et une impulsion dérivée du laser de la photocathode. Deux premières utilisations furent menées sur TESLA-TTF phase pour la photodissociation de cluster, et l'étude de la tenue des optiques à ce rayonnement intense.

Figure 3. Spectre SASE obtenu sur TESLA-TTF phase 1 (Feldhaus). Courbe épaisse : moyenne sur 100 impulsions.

\section{SOURCES LEL IMPLANTÉES SUR ERL}

Afin de disposer avec les LINACs de courants moyens élevés $(100 \mathrm{~mA})$ correspondants à ceux des anneaux de stockage, il faut combiner une technologie LINAC supraconductrice avec la récupération d'énergie. Les électrons accélérés, une fois passés dans la région d'interaction conduisant à la production de rayonnement, sont décélérés en repassent dans le LINAC avec une phase opposée, redonnant ainsi leur énergie à la structure accélératrice. Les ERLs s'affranchissent ainsi des restrictions sur la durée de vie, sur l'émittance et sur la radioprotection. Les idées pionnières, dues à Cornell et à l'Institut Budker (projet MARS) [25], ont conduit aux premières réalisations expérimentales sur CEBAF à Jefferson lab. (Virginie, USA), et sur le LINAC du LEL infra-rouge à $48 \mathrm{MeV}$, permettant une récupération d'énergie de $99 \%$ pour un courant de $5 \mathrm{~mA}$ et $2 \mathrm{~kW}$ de puissance laser moyenne [26]. De même, JAERI-Todai a mis en cuvre la récupération d'énergie, et les tests avec le LEL sont en cours. Des études sont maintenant en cours aux Etats-Unis (Cornell, LBNL), en Angleterre (4GLS combinant un LEL infra-rouge, un oscillateur UV-VUV, un SASE dans le domaine $\mathrm{X}$ mous et des lignes de rayonnement synchrotron), en Allemagne... Des impulsions fs peuvent être obtenues. Il reste à relever de nombreuses difficultés techniques, comme les photo-injecteurs, la stabilité du faisceau, le fort courant moyen...

\section{CONCLUSION}

Dans le cadre des sources cohérentes de lumière basées sur des accélérateurs, plusieurs voies sont possibles pour couvrir le domaine UV à X. Pour le domaine UV, VUV et X mou, les oscillateurs et la génération d'harmoniques cohérentes permettent d'offrir davantage de flexibilité et de très bonnes qualités de sources. Pour atteindre le domaine X autour de $1 \AA$, l'option SASE reste la voie privilégiée, malgré un coût très élevé.

Tableau 1 : Comparaison des sources LEL VUV-soft X ray. Brillance (ph/s/mm2/mrad2/0.1\% Bande passante)

\begin{tabular}{|c|c|c|c|c|}
\hline 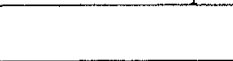 & $\begin{array}{l}\text { LEL sur AS: } \\
\text { oscillateur et GHC }\end{array}$ & $\begin{array}{l}\text { LEL sur Linac : } \\
\text { osciliateur et GHC }\end{array}$ & SASE SUT LINAC & LEL sur ERL \\
\hline Gamme spectrale & UV-VUV $(30 \mathrm{~nm})$ & UV-soft X ray $(10 \mathrm{~nm})$ & $\mathrm{UV-X}(\mathrm{IA})$ & UV-X mou \\
\hline Cadence & $\begin{array}{l}\mathrm{MHz}: \mathrm{O}+\mathrm{GHC} \\
\mathrm{kHz}: \mathrm{Ti}: \mathrm{Sa} \mathrm{GHC}\end{array}$ & $\begin{array}{l}10 \mathrm{~Hz} \text { (macro) } \\
\mathrm{MHz} \text { (micro) }\end{array}$ & $\begin{array}{l}\mathrm{Hz}-\mathrm{kHz} \text { (macro) } \\
\mathrm{MHz} \text { (micro) }\end{array}$ & $\mathrm{GHz}$ \\
\hline Durée d'impulsion & $\begin{array}{l}5 \text { ps O+GHC } \\
\text { loofs:'Ti:Sa GHC }\end{array}$ & ps-fs $\mathrm{O}+\mathrm{GHC}$ & $100 \mathrm{fs}$ & $100 \mathrm{fs}-\mathrm{ps}$ \\
\hline Largeur de raie (\%) & $\begin{array}{l}0.01: \mathrm{O}+\mathrm{GHC} \\
0.1: \text { Ti:Sa GHC }\end{array}$ & $0.01-q q .0 .1$ & $0.1-1$ & 0.1 \\
\hline Cohérence temporelle & $\mathrm{OK}$ & $\mathrm{OK}$ & partielle & $\mathrm{OK}$ \\
\hline Cohérence transverse & OK (cavité optique) & $\mathrm{OK}$ & $\mathrm{OK}$ & OK \\
\hline Stabilité & très bonne & très bonne & mauvaise (jitter,spikes) & \\
\hline Brillance moyenne & $10^{E} 24 / 10^{\mathrm{L}} 21$ & $10^{6} 27-10^{6} 24$ & $10^{2} 24-10^{2} 26$ & $10^{\varepsilon} 25$ \\
\hline Brillance crête & $10^{\prime} 28 / 10^{1} 27$ & $10^{*} 31-10^{*} 28$ & $10^{5} 28 / 10^{5} 34$ & $10^{6} 29$ \\
\hline
\end{tabular}

\section{References}

[1] J. M. J. Madey Phys. Rev. Lett., 36, 717 (1976).

[2] A. Bhowmik, et al; Nucl. Instr. Meth. A296 (1990), 20-24

[3] R. Coisson and F. De Martini, Phys. Quantum Electron. vol 8-9 (Addison-Wesley, Reading; MA) 1982, 939 
[4] K. J. Kim Phys. Rev. Lett. 57 (1986) 1871

[5] G. Neil et al. Phys. Rev. Lett. 84 (4), 662-665 (2000)

[6] M. Billardon, D. Garzella, M.E. Couprie Phys. Rev. Lett. 69, n 16, 19 oct. 1992, 2368-2371

[7] M Trovo' et al, submitted to Nucl. Inst. Meth. A

[8] Yamazaki Tet al., Nucl. Instr. Meth., A331, 27 (1993)

[9] Hama H., Yamazaki J. and Isoyama G. Nucl. Inst. Meth. A 341 (1994) 12-16

[10] V. Litvinenko, S. Park, I. Pinayev, Y. Wu, Nucl. Inst. Meth. A 475 (2001) 195-204

[11] M. E. Couprie, D. Garzella, T. Hara, J. H. Codarbox, M. Billardon, Nucl. Inst. Meth.A 358374 (1995)

[12] , S. Koda, M. Hosaka, M. Katoh, J. Yamazaki, H. Hama, Nucl. Inst. Meth. A 475 (2001) $211-216$

[13] V. Litvinenko et al, SPIE 2988 (1997) 188

[14] M. Hosaka et al. Proced FEL 2001, to be published in Nucl. Inst. Meth. 2002

[15] M. E. Couprie et al Rev. Scient. Inst.; 65 (5), 1994,1485-1495; M. Marsi et al, Appl. Phys. Lett. 70(7) (1997) $895-897$

[16] D. Gracin et al. Nucl. Inst. Meth. A 475 (2001) 635

[17] M. E. Couprie, D. Nutarelli, M. Billardon, Nucl. Inst. Meth. B 144 (1998) 66-74

[18] R. Prazeres et al Europhys. Lett., 4 (7), pp 817-822 (1987)

[19] - G. Dattoli and A. Renieri, Nuovo Cimento, B59, pp1-39, 1980

[20] S. G. Biedron et al, Nucl. Inst. Meth. A Volume 483, Issues 1-2, 94-100

[21] M.J. Hogan et al., Phys. Rev. Lett. 81, 4867 (1998)

[22] S.V. Milton et al., Science 292, 2037 (2001)

[23] V. Ayvazyan et al., Phys. Rev. Lett. 88 n 10 (2002) 11 March 2002; p.104802/1-4. FTXT: Phys Rev Article.

[24] A. Doyuran et al. EPAC 2002, Paris, 3-7 juin 2002

[25] G. N. Kulipanov, A. N. Skrinsky, N. A. Vinokurov Jour. Synchrotron Rad. 1998, 5, 176

[26] G. Neil et al. Phys. Rev. Lett. 84 (4), 662-665 (2000) 